\title{
Sindrome da fragilidade e fatores associados em idosos no pronto atendimento
}

Frailty syndrome and associated factors in the elderly in emergency care

Síndrome de fragilidad y factores relacionados en ancianos en servicio de emergencias

Renata Clemente dos Santos ${ }^{1}$ io https://orcid.org/0000-0003-2916-6832

Rejane Maria Paiva de Menezes² ${ }^{2}$ ic https://orcid.org/0000-0002-0600-0621

Gleicy Karine Nascimento de Araújo ${ }^{3}$ id https://orcid.org/0000-0002-4395-6518

Emanuella de Castro Marcolino² id https://orcid.org/0000-0002-6135-8853

Alana Gonçalves Xavier ${ }^{4}$ ic https://orcid.org/0000-0002-8804-1821

Rafaella Guilherme Gonçalves ${ }^{2}$ io https://orcid.org/0000-0001-8006-8061

Rafaella Queiroga Souto ${ }^{3}$ io https://orcid.org/0000-0002-7368-8497

\section{Como citar:}

Santos RC, Menezes RM, Araújo GK, Marcolino EC, Xavier EG, Gonçalves RG, et al. Síndrome da fragilidade e fatores associados em idosos no pronto atendimento. Acta Paul Enferm. 2020;33:eAPE20190159.

DOI

http://dx.doi.org/10.37689/actaape/2020A00159

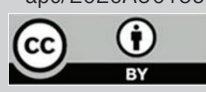

Descritores

Fragilidade; Idoso fragilizado; Enfermagem geriátrica; Enfermagem em emergência; Serviços médicos de emergência

Keywords

Frailty; Frail elderly; Geriatric nursing: Emergence nursing; Emergency medical services

Descriptores Fragilidad; Anciano frágil; Enfermería geriátrica; Enfermería de urgência; Servicios Médicos de urgencia

Submetido 2 de Julho de 2019

Aceito

21 de Outubro de 2019

\section{Autor correspondente}

Rafaella Queiroga Souto

Email: rafaellaqueiroga7@gmail.com

\section{Resumo}

Objetivo: Identificar os fatores associados para a síndrome da fragilidade na pessoa idosa atendida em uma Unidade de Pronto Atendimento.

Métodos: Estudo quantitativo, descritivo, com delineamento de corte transversal, com 146 idosos atendidos em uma Unidade de Pronto Atendimento do interior da Paraíba, nos meses de agosto e setembro de 2017. Foi utilizado um questionário e as escalas de Edmonton Frail Scale e a Hwalek-Sengstock Elder Abuse Screening Test para a caracterização da amostra. A análise dos resultados foi realizada por meio de estatística descritiva (frequência absoluta e relativa, média, mediana, desvio padrão e coeficiente de variação) e inferencial (QuiQuadrado de Pearson; Teste Exato de Fisher e Regressão Logística Múltipla).

Resultados: Os idosos identificados como frágeis são do sexo masculino (58,5\%), acima de 70 anos (80,7\%), sem relacionamento $(47,4 \%)$, alfabetizados $(61,0 \%)$, não trabalham $(54,9 \%)$, residem com filho e cônjuge $(63,2 \%)$, dividem responsabilidades (55,9\%) e têm mais de seis filhos (59,6\%). A área de atendimento que predominou entre os idosos frágeis foi a vermelha (80,0\%), enquanto que a área de caráter da queixa mais prevalente foi a aguda (41,7\%). A maioria deles apresentou risco para a violência $(58,4 \%)$.

Conclusão: A escolaridade, a não execução de atividade laboral, as áreas de classificação e risco para violência são fatores que apresentaram associação com a síndrome da fragilidade, e influenciam no seu desfecho.

\section{Abstract}

Objective: To identify the associated factors for frailty syndrome in elderly people treated in an Emergency Care unit.

Methods: A quantitative, descriptive, cross-sectional study with 146 elderly patients treated in an Emergency Care unit in the inlands of the state of Paraíba in August and September 2017. A questionnaire, the Edmonton Frail Scale and the Hwalek-Sengstock Elder Abuse Screening Test were used for sample characterization. The analysis of results was performed using descriptive statistics (absolute and relative frequency, mean, median, standard deviation and coefficient of variation) and inferential statistics (Pearson's chi-square, Fisher's exact test and multiple logistic regression).

Results: Elderly subjects identified as frail were male (58.5\%), over 70 years old $(80.7 \%)$, with no relationship (47.4\%), literate (61.0\%), not working (54.9\%), living with a child and spouse (63.2\%), share responsibilities (55.9\%) and have more than six children (59.6\%). The red triage area predominated (80.0\%) among frail elderly people, while the most prevalent type of complaint was acute (41.7\%). Most elderly were at risk for violence (58.4\%).

Conclusion: Educational level, not working, classification areas and risk for violence were factors associated with frailty syndrome and influenced its outcome.

\footnotetext{
Universidade Federal do Rio Grande do Norte, Campina Grande, Paraíba, Brasil.

Universidade Federal do Rio Grande do Norte, Natal, Rio Grande do Norte, Brasil.

3Universidade Federal da Paraíba, João Pessoa, PB, Brasil.

4Universidade Estadual da Paraíba, Campina Grande, PB, Brasil.

EUniversidade Federal de Pernambuco, Recife, PE, Brasil.
}

Conflitos de interesse: nada a declarar. 


\section{Resumen}

Objetivo: Identificar los factores relacionados con el síndrome de fragilidad en ancianos en un Servicio de Emergencias.

Métodos: Estudio cuantitativo, descriptivo, de corte transversal, con 146 ancianos atendidos en un Servicio de Emergencias del interior del estado de Paraíba, en los meses de agosto y septiembre de 2017. Se utilizó un cuestionario y las escalas de Edmonton Frail Scale y Hwalek-Sengstock Elder Abuse Screening Test para caracterizar la muestra. El análisis de los resultados se realizó mediante estadística descriptiva (frecuencia absoluta y relativa, promedio, mediana, desviación típica y coeficiente de variación) e inferencial (Prueba $\chi^{2}$ de Pearson, Prueba Exacta de Fisher y Regresión Logística Múltiple).

Resultados: Los ancianos identificados como frágiles son de sexo masculino $(58,5 \%)$, de más de 70 años $(80,7 \%)$, sin una relación afectiva $(47,4 \%)$, alfabetizados (61,0\%), no trabajan (54,9\%), residen con el hijo y su cónyuge $(63,2 \%)$, dividen responsabilidades $(55,9 \%)$ y tienen más de seis hijos $(59,6 \%)$. El área de atención que predominó entre los ancianos frágiles fue la roja $(80,0 \%)$, mientras que el área de carácter de la queja que prevaleció fue aguda (41,7\%). La mayoría presentó riesgo de violencia (58,4\%).

Conclusión: La escolaridad, la no ejecución de actividad laboral, las áreas de clasificación y el riesgo de violencia son factores que presentaron relación con el síndrome de fragilidad e influyeron en el desenlace.

\section{Introdução}

O envelhecimento populacional, associado ao aumento da prevalência de doenças não transmissíveis, pode influenciar o aumento da proporção de multimorbidades, incapacidades e sequelas que exigem açóes integrais do sistema de saúde. ${ }^{(1,2)}$ Além disso, esta parcela populacional apresenta alteraçóes inerentes ao processo de envelhecimento, que potencializam o agravamento desta problemática.

Uma das condiçóes comumente observada, e possível de se apresentar, é a síndrome geriátrica, ${ }^{(1)}$ que se refere à síndrome da fragilidade, de caráter multicausal, podendo se caracterizar pela redução da força muscular e diminuição da reserva fisiológica aumentando, assim, a exposição da pessoa idosa a desfechos adversos, como a dependência física e até a morte. ${ }^{(3)}$

O processo de fragilização envolve a presença de estressores do organismo, limitando, assim, o retorno para a homeostase corporal. $\mathrm{O}$ ciclo que envolve o surgimento da síndrome consiste em uma tríade de desregulação neuroendócrina, sarcopenia e disfunçóes imunológicas, e gera um fenótipo característico composto por perda de peso, exaustão, nível de atividade física, força muscular e lentidão da marcha. A depender das características apresentadas o idoso passa ser classificado em frágil, pré-frágil e não frágil. ${ }^{(4,5)}$

Em estudo realizado em Juiz de Fora - Minas Gerais, com o intuito de avaliar a prevalência e os fatores associados à fragilidade com indivíduos de 65 anos ou mais, obteve-se um número substancial de idosos frágeis, enquanto que metade da amostra estava sob risco de progressão de evoluir para essa condição. Alguns fatores associados identificados neste estudo foram a idade avançada, o comprometimento das atividades básicas de vida e da autopercepção de saúde. ${ }^{(6)}$

Ademais, as fragilidades nas relaçóes familiares, a omissão do poder público e a dependência funcional são potencializadores para ocorrência da violência contra a pessoa idosa. ${ }^{(7,8)}$ Em estudo de base populacional, desenvolvido com 705 idosos, concluiu que a condição de fragilidade apresentou maiores chances para desfechos de violência física e verbal. ${ }^{(9)}$ Entretanto, ainda é incipiente o número de estudos que revelam essa correlação entre o risco para violência e a fragilidade. ${ }^{(10)}$

Acredita-se que a identificação de fatores associados à fragilidade das pessoas idosas trará evidências quanto à necessidade de um acolhimento direcionado e da avaliação multidimensional da pessoa idosa em todos os níveis de atenção à saúde, incluindo as Unidades de Pronto Atendimento (UPA), com o intuito de elaborar uma assistência planejada a fim de minimizar os riscos de desenvolver fragilidade.

Diante do exposto, este estudo segue como pergunta de pesquisa: quais os fatores que influenciam a instalação da síndrome da fragilidade em idosos atendidos em uma Unidade de Pronto Atendimento? Em busca de respostas para tal questionamento, o presente estudo objetivou identificar os fatores associados à síndrome da fragilidade na pessoa idosa atendida em uma Unidade de Pronto Atendimento.

\section{Métodos}

Trata-se de um estudo quantitativo, descritivo, de corte transversal, guiado pelo Strengthening the 
Reporting of Observational Studies in Epidemiology (STROBE). ${ }^{(11)}$ Foi realizado com idosos atendidos em uma UPA do interior da Paraíba, entre agosto e setembro de 2017.

A escolha deste cenário deu-se por considerar que o ambiente é propício à minimização de viés durante a coleta de dados, viabilizando a abordagem diversificada de pacientes polivalentes em clínicas quanto à saúde geral de idosos que ali se dirigem para atendimento, conferindo, assim, uma amostra heterogênea aos graus de instalação da síndrome da fragilidade.

O cálculo da amostra foi realizado com base na média do número de idosos atendidos nos últimos três meses antes da submissão ao comitê de ética, obtendo-se uma população de 1.721 . Foi realizado o cálculo da amostra considerando o erro amostral (e) de 0,08 e nível de confiança de 95\%. Obteve-se uma amostra final constituída por 146 idosos.

Os critérios de inclusão dos sujeitos participantes do estudo foram: pessoas com idade igual ou superior a 60 anos, que deram entrada na UPA, e que estavam em condiçóes estáveis de saúde para responder aos instrumentos de coleta de dados. Essas condições se referem a não estar sob efeitos de sedativos, não apresentar nenhum agravo do seu estado de saúde, que lhe impossibilitava de responder às questóes propostas, e não apresentar qualquer sinal de instabilidade emocional, cognitiva ou física durante a apresentação das questóes propostas nos instrumentos. Foram excluídos aqueles que não apresentavam possibilidades de comunicação.

Para a coleta de informaçóes, aplicou-se o Termo de Consentimento Livre e Esclarecido (TCLE) e utilizou-se a entrevista estruturada através da aplicação pelo pesquisador de um questionário que incluiu variáveis sociodemográficas e algumas questóes sobre sua saúde (levantamento de queixa para o atendimento na UPA). Foram também utilizadas a Escalas de: Edmonton Frail Scale (EFS), ${ }^{(12)}$ de Avaliação da Fragilidade em Idosos. Para o rastreamento do risco da ocorrência da violência contra idosos, utilizou-se a Scale Hwalek-Sengstock Elder Abuse Screening Test (H-S/EAST). ${ }^{(13)}$ Ambas as escalas são validadas e adaptadas transculturalmente para o idioma brasileiro.

A EFS constitui-se por nove domínios e permite classificar a fragilidade em frágil, pré-frágil e não frágil segundo a pontuação dos seus escores já pré-estabelecidos. De zero a quatro, classifica-se como não frágil; entre cinco e seis, como pré-frágil; e sete ou mais, como frágil. ${ }^{(12)}$ Para a análise do presente estudo, a variável foi dicotomizada em frágil e não frágil. Por sua vez, a escala H-S/EAST analisou o risco para violência intrafamiliar através do escore por ela determinado; atribuise um ponto em cada resposta afirmativa, com exceção dos itens um, seis, doze e quatorze, em que o ponto é atribuído à resposta negativa. $\mathrm{Na}$ pontuação maior ou igual a três, ocorre a indicação para o alto risco de o idoso sofrer violência. ${ }^{(13)}$

Definiu-se como variável dependente do estudo, a fragilidade, enquanto que as independentes foram os dados de caracterização (idade, faixa etária, sexo, raça/cor, estado civil, escolaridade, com quem mora, trabalho remunerado, responsabilidade econômica e número de filhos, área de atendimento e queixa principal) e o risco para violência na pessoa idosa.

Para o tratamento dos dados, fez-se uso do SPSS versão 21.0, e a análise dos resultados utilizando a estatística descritiva através de frequência absoluta e relativa, além da média, mediana, desvio padrão e o coeficiente de variação dos sujeitos.

O cruzamento entre a variável dependente e os demais dados foi viabilizado mediante a estatística inferencial através do teste Qui-Quadrado de Pearson ou Teste Exato de Fisher, a depender do número de caselas. Para todas as análises, foi estabelecido o valor de $\mathrm{p}<0,05$ para definição da significância estatística.

Realizou-se ainda uma análise multivariada para avaliação das chances de ocorrência do desfecho da presença de fragilidade. Para tal, foi aplicado o Modelo de Regressão Logística Ajustada por meio do método forward, com intervalo de confiança de $95 \%$ e significância quando $\mathrm{p}<0,05$. Entretanto, para inserção das variáveis independentes na modelagem, foi adotado como critério o valor de $\mathrm{p}<0,02$ nas análises bivariadas.

\section{Resultados}

Quanto às características dos idosos participantes deste estudo ( $n=146)$, a faixa etária variou entre 60 
e 93 anos, com média de 73,35 anos e desvio padrão de 8,45 . No tocante à variável idade, verificou-se um coeficiente de variação de 11,52 e mediana de 72 anos. A variável foi dicotomizada com base na aproximação da mediana, sendo observado que 56,2\% dos idosos estavam na faixa etária de até 70 anos. Em relação à variável sexo, a maioria dos idosos eram homens $(56,2 \%)$, se consideravam pardos $(38,4 \%)$, sem um relacionamento estável $(54,5 \%)$, não trabalhadores $(78,1 \%)$ e não alfabetizados $(71,2 \%)$.

A tabela 1 , mostra a associação entre as variáveis sociodemográficas e a presença de fragilidade entre os idosos. Aqueles com pontuação no escore geral da $\mathrm{EFS}^{(12)}$ menor ou igual a seis pontos foram classificados como não frágeis, e pontuação maior ou igual a sete foram classificados como frágeis. Verificou-se uma associação estatisticamente significativa entre as variáveis escolaridade, trabalho e responsabilidade econômica.

Tabela 1. Associação entre as variáveis sociodemográficas e a presença de fragilidade entre os indivíduos entrevistados

\begin{tabular}{|c|c|c|c|c|}
\hline \multirow[b]{2}{*}{ Variáveis } & \multicolumn{2}{|c|}{ Fragilidade } & \multirow[b]{2}{*}{$\mathrm{p}$-value } & \multirow{2}{*}{$\begin{array}{c}\text { Amostra } \\
\text { válida/em } \\
\text { falta }\end{array}$} \\
\hline & $\begin{array}{c}\text { Frágil } \\
\mathrm{n}(\%)\end{array}$ & $\begin{array}{c}\text { Não frágil } \\
\mathrm{n}(\%)\end{array}$ & & \\
\hline \multicolumn{5}{|l|}{ Sexo } \\
\hline Feminino & $33(52,4)$ & $30(52,4)$ & \multirow[t]{2}{*}{$0,19^{\star}$} & \multirow[t]{2}{*}{$145 / 1$} \\
\hline Masculino & $48(58,5)$ & $34(41,5)$ & & \\
\hline \multicolumn{5}{|l|}{ Idade } \\
\hline Até 70 anos & $35(55,6)$ & $28(44,4)$ & \multirow[t]{2}{*}{$0,67^{\star}$} & \multirow[t]{2}{*}{$146 / 0$} \\
\hline$>70$ anos & $67(80,7)$ & $16(19,3)$ & & \\
\hline \multicolumn{5}{|l|}{ Estado civil } \\
\hline Com relacionamento & $29(43,9)$ & $37(56,1)$ & \multirow[t]{2}{*}{$0,67^{\star}$} & \multirow[t]{2}{*}{$144 / 2$} \\
\hline Sem relacionamento & $37(47,4)$ & $41(52,6)$ & & \\
\hline \multicolumn{5}{|l|}{ Escolaridade } \\
\hline Alfabetizados & $25(61,0)$ & $16(39,0)$ & \multirow[t]{2}{*}{$0,02^{\star}$} & \multirow[t]{2}{*}{$145 / 1$} \\
\hline Não alfabetizados & $42(40,4)$ & $62(59,6)$ & & \\
\hline \multicolumn{5}{|l|}{ Trabalho remunerado } \\
\hline Sim & $5(15,6)$ & $27(84,4)$ & \multirow[t]{2}{*}{$<0,001^{*}$} & \multirow[t]{2}{*}{$145 / 1$} \\
\hline Não & $62(54,9)$ & $51(45,1)$ & & \\
\hline \multicolumn{5}{|l|}{ Mora com quem } \\
\hline Filho e cônjuge & $12(63,2)$ & $7(36,8)$ & \multirow[t]{4}{*}{$0,19^{\star}$} & \multirow[t]{4}{*}{$124 / 22$} \\
\hline Filho & $7(31,8)$ & $15(68,2)$ & & \\
\hline Cônjuge & $16(41,0)$ & $23(59,0)$ & & \\
\hline Outro & $25(56,8)$ & $19(43,2)$ & & \\
\hline \multicolumn{5}{|l|}{ Responsabilidade econômica } \\
\hline Dependem de terceiros & $1(16,7)$ & $5(83,3)$ & \multirow[t]{3}{*}{$0,04^{\star \star}$} & \multirow[t]{3}{*}{$142 / 4$} \\
\hline Mantêm o sustento & $26(38,2)$ & $42(61,8)$ & & \\
\hline Dividem responsabilidades & $38(55,9)$ & $30(44,1)$ & & \\
\hline \multicolumn{5}{|l|}{$N^{0}$ de filhos } \\
\hline Nenhum & $5(45,5)$ & $6(54,5)$ & & \\
\hline Um a três filhos & $11(32,4)$ & $23(67,6)$ & \multirow{3}{*}{$0,07^{*}$} & \multirow{3}{*}{$134 / 12$} \\
\hline Quatro a seis filhos & $20(41,7)$ & $28(58,3)$ & & \\
\hline > seis filhos & $31(59,6)$ & $21(40,9)$ & & \\
\hline
\end{tabular}

*Teste Qui-Quadrado de Pearson; *^Teste Exato de Fisher
Os idosos que foram predominantes na classificação de frágil eram do sexo masculino $(58,5 \%)$, acima de 70 anos (80,7\%), sem relacionamento $(47,4 \%)$, alfabetizados $(61,0 \%)$, não trabalham $(54,9 \%)$, residem com filho e cônjuge $(63,2 \%)$, dividem responsabilidades $(55,9 \%)$ e possuem mais de seis filhos (59,6\%).

No tocante à associação da área de atendimento na UPA (Tabela 2), caráter da queixa, risco para violência e fragilidade, observou-se que todas as variáveis apresentaram associação estatística significativa. A área de atendimento que predominou entre os idosos frágeis foi a vermelha $(80,0 \%)$, enquanto a área de caráter da queixa mais prevalente foi a aguda $(41,7 \%)$, e as pessoas que apresentam risco para violência foram a maioria $(58,4 \%)$.

Tabela 2. Associação entre área de atendimento, caráter da queixa, risco para violência e a presença de fragilidade dos participantes do estudo

\begin{tabular}{|c|c|c|c|c|}
\hline \multirow[b]{2}{*}{ Variáveis } & \multicolumn{2}{|c|}{ Fragilidade } & \multirow[b]{2}{*}{$p$-value } & \multirow{2}{*}{$\begin{array}{c}\text { Amostra } \\
\text { válida/em } \\
\text { falta }\end{array}$} \\
\hline & $\begin{array}{c}\text { Frágil } \\
\mathrm{n}(\%)\end{array}$ & $\begin{array}{c}\text { Não frágil } \\
n(\%)\end{array}$ & & \\
\hline \multicolumn{5}{|c|}{ Área de atendimento na UPA } \\
\hline Verde & $32(34,4)$ & $61(65,6)$ & $<0,001^{\star \star}$ & $145 / 1$ \\
\hline Amarela & $23(62,2)$ & $14(37,8)$ & & \\
\hline Vermelha & $12(80,0)$ & $3(20,0)$ & & \\
\hline \multicolumn{5}{|c|}{ Caráter da queixa } \\
\hline Agudo & $50(41,7)$ & $70(58,3)$ & $0,016^{\star}$ & $145 / 1$ \\
\hline Crônico & $8(32,0)$ & $17(68,0)$ & & \\
\hline \multicolumn{5}{|c|}{ Risco para violência } \\
\hline Com risco & $59(58,4)$ & $42(41,6)$ & $<0,001^{*}$ & $145 / 1$ \\
\hline Sem risco & $8(18,2)$ & $36(81,8)$ & & \\
\hline
\end{tabular}

Entraram no modelo de regressão logística aquelas variáveis que apresentaram valor de $\mathrm{p}<0,02$, a saber: escolaridade, trabalho, número de filhos, área de atendimento, caráter da queixa e risco para violência. Entretanto, as variáveis que permaneceram no modelo foram escolaridade (OR = 3,19; IC95\% $1,21-8,39)$, trabalho $(\mathrm{OR}=8,90$; IC95\% 2,52$31,38)$, área de atendimento amarela $(\mathrm{OR}=2,85$; IC95\% 1,10-7,38); área de atendimento vermelha $(\mathrm{OR}=16,14$; IC95\% 2,56-101,58) e risco para violência $(O R=4,24$; $\mathrm{IC} 95 \% 1,56-11,52)$ (Tabela 3).

Esses achados nos permite inferir que os idosos não alfabetizados e que não trabalham apresentam 3,19 e 8,92 vezes mais chance de ter fragilidade, respectivamente. Os idosos que têm risco para violência têm 4,24 vezes mais chance de ser frágil e re- 
ferente à área de atendimento da UPA, os idosos da área amarela e vermelha exibem 2,85 e 16,14 vezes mais chance de apresentar fragilidade. $\mathrm{O}$ valor de $\mathrm{p}$ para o teste de Hosmer e Lemeshow foi de 0,910, de modo que o p-valor maior que 0,05 indica que há indícios suficientes para a aceitação do modelo.

A área baseada na curva Receiver Operating Characteristics (ROC) para o modelo de regressão logística acima foi de 0,81 (IC 0,74-0,88; p<0,001) para presença da fragilidade, com discriminação excelente, conforme observamos na figura 1 .

Tabela 3. Variáveis associadas à presença de fragilidade por meio de regressão logística ajustada entre idosos atendidos em UPA

\begin{tabular}{l|c|c|c}
\hline Variáveis & OR & IC & $p$-value \\
\hline Escolaridade & & & \\
$\quad$ Alfabetizado & 1,00 & - & - \\
$\quad$ Não alfabetizado & 3,19 & {$[1,21-8,39]$} & 0,019 \\
Trabalho remunerado & & & \\
$\quad$ Sim & 1,00 & - & - \\
$\quad$ Não & 8,90 & {$[2,52-31,38]$} & 0,001 \\
\hline Área de atendimento na UPA & & & \\
$\quad$ Verde & 1,00 & - & - \\
$\quad$ Amarela & 2,85 & {$[1,10-7,38]$} & 0,030 \\
$\quad$ Vermelha & 16,14 & {$[2,56-101,58]$} & 0,003 \\
Risco para violência & & & \\
$\quad$ Sem risco & 1,00 & - & - \\
$\quad$ Com risco & 4,24 & {$[1,56-11,52]$} & 0,005 \\
\hline
\end{tabular}

$\mathrm{R}^{2}$ ajustado: 0,416; OR - Odds Ratio; IC - intervalo de confiança; *Significância do teste

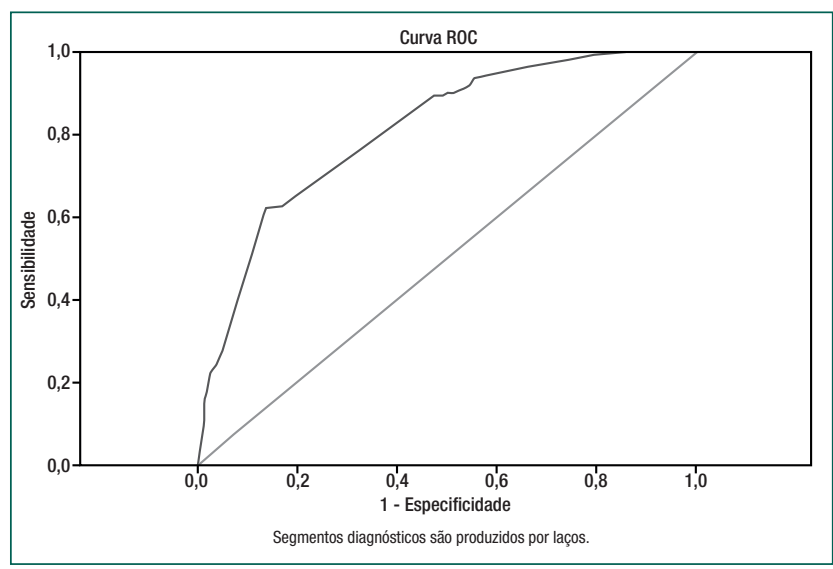

Figura 1. Curva ROC com base no modelo de regressão logística da presença da fragilidade entre idosos atendidos na UPA

\section{Discussão}

No presente estudo, a população masculina apresentou maior prevalência de síndrome da fragilidade. Essa informação diverge da maior parte dos estudos, ${ }^{(14-17)}$ os quais abordam o fenômeno de feminização da velhice e a maior prevalência da fragilidade nesta população embasados na condição de que a população feminina possui maior expectativa de vida, menores taxas de mortalidade por causas externas, menor exposição a riscos ocupacionais, menor consumo de tabaco e álcool e maior procura por serviços de saúde em comparação aos homens. Consequentemente, a populaçáo de idosas seria mais acometida pela síndrome da fragilidade por experienciar mais as alteraçóes do envelhecimento, ao alcançar idade maior que os homens.

Os dados identificaram que $80,7 \%$ dos idosos acima de 70 anos de idade apresentavam a síndrome da fragilidade, enquanto que, para os idosos com idade inferior a 70 anos, essa condição estava presente em 55,6\%. Estudos nacionais e internacionais desenvolvidos com a pessoa idosa obtiveram resultados semelhantes. ${ }^{(14,17,18)}$

$\mathrm{O}$ avançar da idade tende a se relacionar à deterioração das capacidades funcionais e ao aparecimento de doenças não transmissíveis, sendo, então, potencializadores para o surgimento da dependência funcional na execução das atividades de vida diária. A fragilidade mostra-se como uma síndrome progressiva fundamentada nas mudanças fisiológicas e patológicas e no declínio dos sistemas refletindo diretamente na funcionalidade do idoso. ${ }^{(19-21)}$

Com relação ao estado civil, prevaleceram idosos sem relacionamento. Esse achado corrobora com a literatura nacional. ${ }^{(16)}$ A falta ou diminuição das relaçóes sociais podem levar à síndrome da fragilidade na pessoa idosa, uma vez que esta seria socialmente menos ativa. ${ }^{(16)}$

No que diz respeito à escolaridade, os idosos não alfabetizados têm mais chance de desenvolver a síndrome da fragilidade, convergindo da literatura, a qual evidencia que há maior predomínio de síndrome da fragilidade em grupos populacionais que possuem um nível educacional mais baixo. ${ }^{(16,17)}$

Foi identificada associação entre a síndrome da fragilidade em idosos que não trabalham. Esta informação é compatível com registros da literatura, ${ }^{(22,23)}$ uma vez que idosos que permanecem ativos têm melhor desempenho da função cognitiva e autonomia nas atividades diárias. 
Na relação entre a queixa do idoso para internação no serviço de saúde e a fragilidade do mesmo, o presente estudo demonstrou que $41,7 \%$ dos idosos entrevistados apresentaram queixas de caráter agudo, e tal situação demonstrou associação com a síndrome da fragilidade $(\mathrm{p}=0,01)$. Entretanto, um estudo internacional demonstra que a fragilidade se encontra diretamente relacionada com morbidades crônicas e o aumento progressivo da idade. ${ }^{(24)}$

A associação entre a queixa aguda do idoso e a síndrome da fragilidade pode ser explicada pela característica do serviço de saúde locus da pesquisa, UPA, em que predominam as queixas de caráter agudo. Essas queixas em pessoas vulneráveis surgem devido aos déficits nos processos fisiológicos de reparação dos pequenos estressores. Nesse contexto, pessoas frágeis estão sujeitas a complicaçóes agudas de doenças crônicas caracterizando o quadro dos idosos investigados. ${ }^{(25)}$

Em relação à classificação de risco para o atendimento de usuários nas UPA, utiliza-se o Protocolo de Manchester, o qual destina a área vermelha para o usuário com necessidade de assistência emergente com risco iminente de morte. Trata-se de uma área onde se executam procedimentos especiais e invasivos. Já a área amarela destina-se a pacientes estabilizados, classificados como semicríticos, e o atendimento na área verde corresponde ao espaço delimitado ao recebimento de pacientes que precisam de observação. ${ }^{(26,27)}$

Outro elemento significativo trata-se da associação direta entre risco de sofrer violência com ser frágil entre os idosos. A violência intrafamiliar tem se mostrado como a maior produtora do risco de violência para o idoso, o que potencializa a instalação da síndrome da fragilidade no mesmo. Estudos brasileiros ressaltaram que a condição da fragilidade encontra-se associada a maiores razóes de chances entre a violência física e/ou verbal, ou somente violência física ou violência verbal. ${ }^{(9)}$

Além da associação entre as variáveis dependente e independentes, existe maior chance de ocorrência da fragilidade quando se considera escolaridade, renda, risco para violência e tipo de atendimento na UPA. Assim, a regressão logística evidenciou maior chance para fragilidade de idosos não alfabetizados, em consonância com estudo brasileiro sobre fatores de risco associados à fragilidade de idosos, que destacou os idosos com menor escolaridade (68\%), menor renda $(64 \%)$ e maior número de comorbidades $(77 \%)$ diretamente associados à fragilidade. ${ }^{(28)}$

Um estudo asiático observou no modelo multivariado que estar no nível mais baixo de educação aumentou o risco relativo de ser pré-frágil, em comparação com o não frágil em idosos. Nunca ter sido empregado ou ter tido uma ocupação pouco qualificada aumentou o risco relativo de ser frágil, comparando-se com ser não frágil. ${ }^{(29)}$

Entende-se que idosos com condiçóes clínicas mais graves, ou seja, aqueles que se encontram internados na área vermelha de UPA, apresentam um estado catabólico que diminui as reservas corporais, o que contribui para a fragilidade dos mesmos, independentemente da idade e do status funcional pré-hospitalar. Assim, a inserção no serviço de saúde em uma situação de maior gravidade por si só já potencializa a chance do idoso ser frágil, ${ }^{(30)}$ bem como a própria hospitalização se mostra como variável associada à fragilidade. ${ }^{(31)}$

Nessa perspectiva, entende-se que o cuidado a idosos frágeis deve inserir a investigação direcionada a situaçôes de violência. Ademais, o diagnóstico precoce de situaçôes de violência pode favorecer medidas protetivas voltadas à síndrome da fragilidade e à ocorrência de violência contra a pessoa idosa.

Com base nos dados apresentados, entende-se que a presença da fragilidade no idoso relaciona-se com o contexto social e econômico no qual encontra-se inserido. Desse modo, intervençôes com vista à redução ou minimização da progressão da fragilidade na população idosa incluem fatores comportamentais, como qualidade de vida, engajamento social e suporte familiar. ${ }^{(32)}$

O delineamento deste estudo se caracteriza por apresentar um corte transversal e, consequentemente, não possibilitar avaliar a causalidade e nem a longitudinalidade do fenômeno em questão. Deste modo, é capaz de realizar associaçóes e comparaçôes, apontando possíveis de fatores de risco.

\section{Conclusão}

Dentre os idosos estudados, a fragilidade predominou em indivíduos homens, com mais de 70 anos, 
sem relacionamento, alfabetizados, que exercem alguma atividade laboral, moram com alguém e têm mais de seis filhos. No tocante à área de atendimento e de caráter da queixa, prevaleceram entre os idosos frágeis a área vermelha e de caráter agudo. Ademais, o risco para violência também esteve associado à fragilidade, uma vez que os indivíduos com risco para violência se configuraram com maiores percentuais da síndrome. A associação destas variáveis foi confirmada pelo modelo de regressão, haja vista que permaneceram no modelo final as variáveis escolaridade, trabalho, áreas de atendimento amarela e vermelha e o risco para violência.

\section{Agradecimentos}

Ao Conselho Nacional de Desenvolvimento Científico e Tecnológico $(\mathrm{CNPq})$ pela concessão da Bolsa de Iniciação Científica de acordo com o número de identificação do projeto 16048586 .

\section{Colaborações}

Santos RC, Menezes RMP, Araújo GKN, Marcolino EC, Xavier AG, Gonçalves RG declaram que contribuíram com a concepção do manuscrito, análise e interpretação dos dados, redação do artigo, revisão crítica relevante do conteúdo intelectual e aprovação da versão final a ser publicada.

\section{Referências}

1. World Health Organization (WHO). World report on ageing and health [Internet]. Genève: WHO; 2015. [cited 2019 Oct 19]. Available from: https://www.who.int/ageing/publications/worldreport-2015/en/

2. Miranda GM, Mendes ACG, Silva AL. Population aging in Brazil: current and future social challenges and consequences. Rev Bras Geriatr Gerontol. 2016;19(3):507-19.

3. Morley JE, Vellas B, Van Kan GA, Anker SD, Bauer JM, Bernabei $\mathrm{R}$, et al. Frailty consensus: a call to action. J Am Med Dir Assoc. 2013;14(6):392-97.

4. Fried LP, Tangen CM, Walston J, Newman AB, Hirsch C, Gottdiener J, et al. Frailty in older adults: evidence for a phenotype. J Gerontol A Biol Sci Med Sci. 2001;56(3):146-57.
5. Walston J, Hadley EC, Ferrucci L, Guralnik JM, Newman AB, Studenski SA, et al. Research agenda for frailty in older adults: toward a better understanding of physiology and etiology: summary from the American Geriatrics Society/National Institute on Aging Research Conference on Frailty in Older Adults. J Am Geriatr Soc. 2006;54(6):991-1001.

6. Lourenço RA, Moreira VG, Banhato EF, Guedes DV, Silva KC, Delgado $\mathrm{FE}$, et al. Prevalência e fatores associados à fragilidade em uma amostra de idosos que vivem na comunidade da cidade de Juiz de Fora, Minas Gerais, Brasil: estudo FIBRA-JF. Ciênc Saúde Colet. 2019 ;24(1):35-44.

7. Rocha RC, Côrtes MC, Dias EC, Gontijo ED. Violência velada e revelada contra idosos em Minas Gerais-Brasil: análise de denúncias e notificações. Saúde Debate. 2018; 42(Esp)4:81-94.

8. Paraíba PM, Silva MC. Perfil da violência contra a pessoa idosa na cidade do Recife-PE. Rev Bras Geriatr Gerontol. 2015;18(2):295-306.

9. Tavares DM, Belisário MS, Dias FA, Pegorari M, Mapelli M, Ferreira PC. Association between the risk of violence against the elderly person and the frailty syndrome. Innov Aging. 2017;1(Suppl 1):382-83.

10. Santos RC, Menezes RM, Gonçalves RG, Silva JC, Almeida JL, Araújo GK. Violence and frailty in the elderly. Rev Enferm UFPE Online. 2018;12(8):2227-34.

11. Cheng A, Kessler D, Mackinnon R, Chang TP, Nadkarni VM, et al. Reporting guidelines for health care simulation research: extensions to the CONSORT and STROBE statements. Simul Healthcare. 2016;11(4):238-48.

12. Hwalek MA, Sengstock MC. Assessing the probability of abuse of the elderly: toward development of a clinical screening instrument. J Appl Gerontol. 1986;5(2):153-73.

13. Neale AV, Hwalek MA, Scott RO, Sengstock MC, Stahl C. Validation of the Hwalek-Sengstock elder abuse screening test. J Appl Gerontol. 1991;10(4):406-18

14. Jesus IT, Orlandi AA, Grazziano ED, Zazzetta MS. Frailty of the socially vulnerable elderly. Acta Paul Enferm. 2017;30(6):614-20.

15. Gross CB, Kolankiewicz AC, Schmidt CR, Berlezi EM. Frailty levels of elderly people and their association with sociodemographic characteristics. Acta Paul Enferm. 2018;31(2):209-16.

16. Duarte M, Paúl C. Prevalence of phenotypic frailty during the aging process in a Portuguese community. Rev Bras Geriatr Gerontol. 2015;18(4):871-80.

17. Rodrigues RA, Fhon JR, Pontes MD, Silva AO Haas VJ, Santos JL. Frailty syndrome among elderly and associated factors: comparison of two cities. Rev Lat Am Enferm. 2018;26:e3100.

18. Hajek A, Brettschneider C, Posselt T, Lange C, Mamone S, Wiese B. et al. Predictors of frailty in old age-results of a longitudinal study. J Nutr Health Aging. 2016 ;20(9):952-57.

19. Carneiro JA, Cardoso RR, Durães MS, Guedes MC, Santos FL, Costa $\mathrm{FM}$, et al. Frailty in the elderly: prevalence and associated factors. Rev Bras Enferm. 2017;70(4):780-5.

20. Jesus IT, Orlandi AA, Grazziano ES, Zazzetta MS. Frailty of the socially vulnerable elderly. Acta Paul Enferm. 2017;30(6):614-20.

21. Tavares DM, Almeida EG, Ferreira PC, Dias FA, Pegarari MS. Fragility status among elderly with indicative of depression by gender. J Bras Psiquiatr. 2014; 63 (4):347-53.

22. Melo EM, Marques AP, Leal MC, Melo HM. Frailty syndrome and associated factors in elderly residents in long-term institutions. Saúde Debate. 2018 [; 42(117):468-80. 
23. Amorim JS, Salla S, Trelha CS. Factors associated with work ability in the elderly: systematic review. Rev Bras Epidemiol. 2014;17(4):830-41.

24. Orkaby AR, Onuma O, Qazi S, Gaziano JM, Driver JA. Preventing cardiovascular disease in older adults: One size does not fit all. Cleve Clin J Med. 2018;85(1):55-64.

25. Mudge AM, McRae P, Hubbard RE, Peel NM, Lim WK, Barnett AG, Inouye SK. Hospital-associated complications of older people: a proposed multicomponent outcome for acute care. J Am Geriatr Soc. 2019;67(2):352-56.

26. Oliveira JL, Gatti AP, Barreto MS, Bellucci Junior JA, Góes HL, Matsuda LM. User embracement with risk classification: perceptions of the service users of an emergency care unit. Texto Contexto Enferm. 2017;26(1):e0960014.

27. Andrade LA, Santos SD, Corpolato RC, Willig MH, Mantovani MD, Aguilera AL. Elderly care in the emergency department: an integrative review. Rev Bras Geriatr Gerontol. 2018;21(2):243-53.
28. Lourenço RA, Moreira VG, Banhato EF, Guedes DV, Silva KC, Delgado $\mathrm{FE}$, Marmora $\mathrm{CH}$. Prevalence of frailty and associated factors in a community-dwelling older people cohort living in Juiz de Fora, Minas Gerais, Brazil: Fibra-JF Study. Ciênc. Saúde Colet. 2019;(1):35-44.

29. Siriwardhana DD, Weerasinghe MC, Rait G, Falcaro M, Scholes S, Walters KR. Prevalence of frailty in rural community-dwelling older adults in Kegalle district of Sri Lanka: a population-based crosssectional study. BMJ Open. 2019; 9(1):e026314.

30. Joseph B, Jehan FS. The mobility and impact of frailty in the intensive care unit. Surg Clin. 2017;97(6):1199-213.

31. Carneiro JA, Cardoso RR, Durães MS, Guedes MC, Santos FL, Costa FM, Caldeira AP. Frailty in the elderly: prevalence and associated factors. Rev Bras Enferm. 2017;70(4):747-52.

32. Walston J, Buta B, Xue QL. Frailty screening and interventions: considerations for clinical practice. Clin Geriatr Med. 2018;34(1):2538. 